ANNALES

POLONICI MATHEMATICI

$81.1(2003)$

\title{
On the Euler characteristic of the link of a weighted homogeneous mapping
}

\author{
by Piotr Dudziński (Gdańsk)
}

\begin{abstract}
The paper is concerned with an effective formula for the Euler characteristic of the link of a weighted homogeneous mapping $F: \mathbb{R}^{n} \rightarrow \mathbb{R}^{k}$ with an isolated singularity. The formula is based on Szafraniec's method for calculating the Euler characteristic of a real algebraic manifold (as the signature of an appropriate bilinear form). It is shown by examples that in the case of a weighted homogeneous mapping it is possible to make the computer calculations of the Euler characteristics much more effective.
\end{abstract}

Let $F: \mathbb{R}^{n} \rightarrow \mathbb{R}^{k}$, where $n>k+1$, be a polynomial mapping and let $L=\left\{x \in S^{n-1} \mid F(x)=0\right\}$. Let $\chi(L)$ denote the Euler characteristic of $L$. There is an algebraic formula, due to Szafraniec [7], which expresses $\chi(L)$ in terms of the signature of an appropriate bilinear form (provided that $L$ is smooth).

The aim of this paper is to show that in the weighted homogeneous case the general formula for the Euler characteristic of $L$ can be replaced by a simpler one. It makes practical (computer) calculations of the Euler characteristic more effective. Actually, in some cases the general method is ineffective because of huge computations.

The basic idea is to consider the intersection of $F^{-1}(0)$ with the hyperplanes $\left\{x_{n}=0\right\}$ and $\left\{x_{n}=1\right\}$. We prove that the Euler characteristic of $L$ is determined by the behavior of $F$ on these spaces, so the formula for $\chi(L)$ becomes simpler (we consider only the case when the manifold $L$ is of even dimension, for otherwise its Euler characteristic is zero).

We also consider the simpler invariant $\chi(L) / 2(\bmod 2)$; we give a simple formula which requires only calculating the dimension of an appropriate algebra (instead of computing a signature).

There is a computer program by Andrzej Eecki to compute the dimensions of quotient algebras and signatures of bilinear forms on these algebras;

2000 Mathematics Subject Classification: 14P05, 57R70.

Key words and phrases: weighted homogeneous mapping, Euler characteristic. 
however, reducing the dimension of the domain of $F$ makes the use of the program considerably more effective.

Let $d_{1}, \ldots, d_{k}, w_{1}, \ldots, w_{n}$ be positive integers. For $x=\left(x_{1}, \ldots, x_{n}\right) \in \mathbb{R}^{n}$ and $\lambda \in \mathbb{R}$ we write $\lambda . x=\left(\lambda^{w_{1}} x_{1}, \ldots, \lambda^{w_{n}} x_{n}\right)$ and $x^{\prime}=\left(x_{1}, \ldots, x_{n-1}\right) \in$ $\mathbb{R}^{n-1}$, so $x=\left(x^{\prime}, x_{n}\right) \in \mathbb{R}^{n}$.

Let $F: \mathbb{R}^{n} \rightarrow \mathbb{R}^{k}$ be a polynomial mapping such that every $F_{i}$ is a weighted homogeneous polynomial of degree $d_{i}$ with respect to the weights $\operatorname{wt}\left(x_{j}\right)=w_{j}$, i.e. $F_{i}(\lambda . x)=\lambda^{d_{i}} F_{i}(x)$ for every $x \in \mathbb{R}^{n}, \lambda \in \mathbb{R}$.

Renumbering the variables if necessary, we may assume that $w_{n}$ is odd (there is at least one odd weight). Let $c$ be the smallest positive integer such that each $w_{j}$ divides $c$. Let $a_{j}=c / w_{j}$ and $\omega(x)=\sum_{j=1}^{n} x_{j}^{2 a_{j}} /\left(2 a_{j}\right)$. Put $\Sigma_{r}=\left\{x \in \mathbb{R} \mid \omega(x)=r^{2 c}\right\}, \Gamma_{r}=\left\{x \in \mathbb{R} \mid \omega(x) \leq r^{2 c}\right\}$ and $L_{r}=$ $F^{-1}(0) \cap \Sigma_{r}$. Note that if $x \in L_{r}$ then $\lambda . x \in L_{\lambda r}$ for every $\lambda \neq 0$.

Write $G\left(x^{\prime}\right)=F\left(x^{\prime}, 1\right)$ and $H\left(x^{\prime}\right)=F\left(x^{\prime}, 0\right)$ for every $x^{\prime} \in \mathbb{R}^{n-1}$. Assume that

$$
\begin{array}{ll}
\operatorname{rank}\left[D G\left(x^{\prime}\right)\right]=k & \text { for every } x^{\prime} \in G^{-1}(0) \\
\operatorname{rank}\left[D H\left(x^{\prime}\right)\right]=k & \text { for every } x^{\prime} \in H^{-1}(0)-0 .
\end{array}
$$

As a consequence of (1) and (2) we obtain

LEMMA 1. $\operatorname{rank}[D F(x)]=k$ for every $x \in F^{-1}(0)-0$ and $F^{-1}(0)$ meets each $\Sigma_{r}$ transversally.

Proof. Suppose that $\operatorname{rank}[D F(x)]<k$ for some $x \in F^{-1}(0), x \neq 0$. Clearly, $D_{j} H\left(x^{\prime}\right)=D_{j} F\left(x^{\prime}, 0\right)$ for $j=1, \ldots, n-1$, hence if $x_{n}=0$ then $\operatorname{rank}\left[D H\left(x^{\prime}\right)\right]<k$.

Assume that $x_{n} \neq 0$. Each $F_{i}$ is a weighted homogeneous polynomial, hence it satisfies the Euler formula:

$$
\sum_{j=1}^{n} w_{j} x_{j} D_{j} F_{i}(x)=d_{i} F_{i}(x)
$$

for $i=1, \ldots, k$. In particular, if $x_{n} \neq 0$, then the last column of the matrix $[D F(x)]$ is a linear combination of the $n-1$ preceding columns. Again by weighted homogeneity of each $F_{i}$ we have

$$
\lambda^{w_{j}} D_{j} F_{i}(\lambda . x)=\lambda^{d_{i}} D_{j} F_{i}(x)
$$

and thus

$$
D_{j} F_{i}(\lambda . x)=\lambda^{d_{i}-w_{j}} D_{j} F_{i}(x) .
$$

Therefore, $\operatorname{rank}[D F(\lambda . x)]=\operatorname{rank}[D F(x)]$ for $\lambda \neq 0$. Then, letting $\lambda=$ $x_{n}^{-1 / w_{n}}$ we have $\lambda . x \in F^{-1}(0) \cap\left\{x_{n}=1\right\}=G^{-1}(0)$ and $\operatorname{rank}\left[D G\left(x^{\prime}\right)\right]<k$, where $\lambda . x=\left(x^{\prime}, 1\right)$, which proves the first claim. 
In order to prove the second assertion of the lemma, we shall show that the rank of the matrix

$$
M=\left[\begin{array}{ccc}
D_{1} \omega(x) & \ldots & D_{n} \omega(x) \\
D_{1} F_{1}(x) & \ldots & D_{n} F_{1}(x) \\
& \ldots & \\
D_{1} F_{k}(x) & \ldots & D_{n} F_{k}(x)
\end{array}\right]
$$

is $k+1$ for every $x \in F^{-1}(0) \cap \Sigma_{r}$. We may assume that $x_{1} \neq 0$. Let us multiply the first column of $M$ by $w_{1} x_{1}$ and then add to it a linear combination of the form $\sum_{j=2}^{n} w_{j} x_{j} A_{j}$, where $A_{j}$ denotes the $j$ th column of $M$. Then the first column becomes

$$
\left[\begin{array}{c}
\sum_{j=1}^{n} w_{j} x_{j} D_{j} \omega(x) \\
\sum_{j=1}^{n} w_{j} x_{j} D_{j} F_{1}(x) \\
\cdots \\
\sum_{j=1}^{n} w_{j} x_{j} D_{j} F_{k}(x)
\end{array}\right] .
$$

Again, by the Euler formula we have

$$
\sum_{j=1}^{n} w_{j} x_{j} D_{j} F_{i}(x)=d_{i} F_{i}(x)
$$

for $i=1, \ldots, k$ and

$$
\sum_{j=1}^{n} w_{j} x_{j} D_{j} \omega(x)=2 c \omega(x),
$$

so the first column has the form

$$
\left[\begin{array}{c}
2 c r^{2 c} \\
0 \\
0 \\
\cdots \\
0
\end{array}\right]
$$

Thus the first row of $M$ cannot be written as a linear combination of the remaining rows. So the first part of the lemma yields the assertion.

Therefore each $L_{r}$ is a smooth $(n-k-1)$-dimensional compact manifold and $\chi\left(L_{r}\right)=\chi(L)$ (the independence of the link from the function $\omega$ has been proven in [3]; in our case it may be observed that $L$ and $L_{r}$ are homeomorphic by flowing along a vector field tangent to $F^{-1}(0)$ ). The Euler characteristic of every compact odd-dimensional manifold is zero, so from now on we shall assume that $n-k$ is odd.

Note that $H$ is also weighted homogeneous, hence the same reasoning shows that $H^{-1}(0)$ meets each $\widetilde{\Sigma}_{r}=\Sigma_{r} \cap\left\{x_{n}=0\right\}$ transversally and

$$
\chi\left(H^{-1}(0) \cap \widetilde{\Sigma}_{r}\right)=0 .
$$

We shall denote by $\pi$ the projection on the $n$th coordinate in $\mathbb{R}^{n}$. 
Lemma 2. For sufficiently large $r$,

$$
\chi\left(L_{r} \cap\left\{-1 \leq x_{n} \leq 1\right\}\right)=0 .
$$

Proof. It is easy to check (by the same method as in the proof of Lemma 1) that the rank of the matrix

$$
\left[\begin{array}{cccc}
D_{1} \pi(x) & \ldots & D_{n-1} \pi(x) & D_{n} \pi(x) \\
D_{1} \omega(x) & \ldots & D_{n-1} \omega(x) & D_{n} \omega(x) \\
D_{1} F_{1}(x) & \ldots & D_{n-1} F_{1}(x) & D_{n} F_{1}(x) \\
& \ldots & & \\
D_{1} F_{k}(x) & \ldots & D_{n-1} F_{k}(x) & D_{n} F_{k}(x)
\end{array}\right]
$$

is $k+2$ for every $x \in L_{1} \cap\left\{x_{n}=0\right\}$. This means that $\pi_{\mid L_{1}}$ has no critical points in the hyperplane $\left\{x_{n}=0\right\}$ (see [4]). Hence for small $\varepsilon>0, \pi_{\mid L_{1}}$ has no critical points in the set $L_{1} \cap\left\{-\varepsilon \leq x_{n} \leq \varepsilon\right\}$.

So $L_{1} \cap\left\{x_{n}=0\right\}$ is a deformation retract of $L_{1} \cap\left\{-\varepsilon \leq x_{n} \leq \varepsilon\right\}$, and then by (3), $\chi\left(L_{1} \cap\left\{-1 \leq x_{n} \leq 1\right\}\right)=0$. The proof is completed by observing that if $r=\varepsilon^{-1 / w_{n}}$, then $x \mapsto r . x$ maps homeomorphically the set $L_{1} \cap\left\{-\varepsilon \leq x_{n} \leq \varepsilon\right\}$ onto $L_{r} \cap\left\{-1 \leq x_{n} \leq 1\right\}$.

Theorem 3. $\chi(L)=2 \chi\left(F^{-1}(0) \cap\left\{x_{n}=1\right\}\right)$.

Proof. Clearly,

$$
\begin{aligned}
\chi(L)= & \chi\left(L_{r}\right) \\
= & \chi\left(L_{r} \cap\left\{-1 \leq x_{n} \leq 1\right\}\right)+\chi\left(L_{r} \cap\left\{x_{n} \geq 1\right\}\right) \\
& +\chi\left(L_{r} \cap\left\{x_{n} \leq-1\right\}\right)-\chi\left(L_{r} \cap\left\{x_{n}= \pm 1\right\}\right) .
\end{aligned}
$$

By Lemma 2, $\chi\left(L_{r} \cap\left\{-1 \leq x_{n} \leq 1\right\}\right)=0$ for large $r$. Using similar arguments to those above we can prove that for large $r, L_{r}$ meets $\left\{x_{n}= \pm 1\right\}$ transversally and then $\chi\left(L_{r} \cap\left\{x_{n}= \pm 1\right\}\right)=0$.

If $x \in L_{r} \cap\left\{x_{n} \geq 1\right\}$ then set $\gamma(x)=x_{n}^{-1 / w_{n}}$. Then $x \mapsto \gamma(x) . x$ maps $L_{r} \cap\left\{x_{n} \geq 1\right\}$ homeomorphically onto the set $F^{-1}(0) \cap\left\{x_{n}=1\right\} \cap \Gamma_{r}$, which for large $r$ is a deformation retract of $F^{-1}(0) \cap\left\{x_{n}=1\right\}=G^{-1}(0)$. Moreover, the map $x \mapsto(-1) \cdot x$ is a homeomorphism between $L_{r} \cap\left\{x_{n}=1\right\}$ and $L_{r} \cap\left\{x_{n}=-1\right\}$ (recall that $w_{n}$ is odd), so the proof is complete.

Let $I(H) \subset \mathbb{R}\left[x^{\prime}\right]=\mathbb{R}\left[x_{1}, \ldots, x_{n-1}\right]$ be the ideal generated by $H_{1}, \ldots, H_{k}$ and all $(k+1) \times(k+1)$-minors $\partial\left(\omega, H_{1}, \ldots, H_{k}\right) / \partial\left(x_{j_{1}}, \ldots, x_{j_{k+1}}\right)$.

If $\operatorname{dim} \mathbb{R}\left[x^{\prime}\right] / I(H)<\infty$ then there are a finite number of singular points of $H^{-1}(0)$ and of critical points of $\omega$ restricted to the smooth part of $H^{-1}(0)$. But the map $H$ is weighted homogeneous, hence $H^{-1}(0)$ is transversal to each $\widetilde{\Sigma}_{r}$, so there are no critical points of $\omega$ on the smooth part of $H^{-1}(0)$. It follows by the same method as in the proof of Lemma 1 that if $\left(x_{1}, \ldots, x_{n-1}\right) \in H^{-1}(0)$ is a singular point of $H^{-1}(0)$, then for every 
$\lambda \neq 0,\left(\lambda^{w_{1}} x_{1}, \ldots, \lambda^{w_{n-1}} x_{n-1}\right)$ is also a singular point of $H^{-1}(0)$. Thus we have proved

Lemma 4. If $\operatorname{dim} \mathbb{R}\left[x^{\prime}\right] / I(H)<\infty$ then $\operatorname{rank}\left[D H\left(x^{\prime}\right)\right]=k$ for every $x^{\prime} \in H^{-1}(0)-\{0\}$.

There is an algebraic formula, due to Szafraniec [7], which expresses the Euler characteristic of an algebraic manifold as the signature of an appropriate bilinear form. There is also an algebraic method for verifying assumption (1).

Assume that $\operatorname{dim} \mathbb{R}\left[x^{\prime}\right] / I(G)=d<\infty$. According to [7], there is a certain (explicitly defined) linear functional $\phi: \mathbb{R}\left[x^{\prime}\right] / I(G) \rightarrow \mathbb{R}$. Set $M=$ $\partial\left(G_{1}, \ldots, G_{k}\right) / \partial\left(x_{1}, \ldots, x_{k}\right)$ and define a bilinear form $\Phi_{M}: \mathbb{R}\left[x^{\prime}\right] / I(G) \times$ $\mathbb{R}\left[x^{\prime}\right] / I(G) \rightarrow \mathbb{R}$ by $\Phi_{M}(f, g)=\phi(M f g)$.

Theorem 5. Assume that $\Phi_{M}$ is non-degenerate. Then $G^{-1}(0)$ is a smooth manifold and $\chi\left(G^{-1}(0)\right)=$ signature $\Phi_{M}$.

For the proof see [7].

Therefore we have

Theorem 6. Assume that $\operatorname{dim} \mathbb{R}\left[x^{\prime}\right] / I(H)<\infty$ and $\operatorname{dim} \mathbb{R}\left[x^{\prime}\right] / I(G)<$ $\infty$ and that $\Phi_{M}$ is non-degenerate. Then $\chi(L)=2 \cdot$ signature $\Phi_{M}$.

Since $\Phi_{M}$ is non-degenerate, signature $\Phi_{M} \equiv \operatorname{dim} \mathbb{R}\left[x^{\prime}\right] / I(G)(\bmod 2)$, hence we obtain a simpler formula for the simpler invariant $\chi(L) / 2(\bmod 2)$ :

COROLlary 7. Under the assumptions of Theorem 6 ,

$$
\chi(L) / 2 \equiv \operatorname{dim} \mathbb{R}\left[x^{\prime}\right] / I(G)(\bmod 2) .
$$

In [2] another formula is given which expresses $\chi(L) / 2(\bmod 2)$ in terms of the dimensions of appropriate local algebras in the case of $F: \mathbb{R}^{n} \rightarrow \mathbb{R}$ with a non-isolated singularity.

EXAMPle 1. Let $F(x, y, z, t)=x^{2} z t+t^{2}+x^{5} y z+y^{2} z^{2}+x^{3} y+x^{2} y^{2}+$ $x y^{3}+y^{5}$. The polynomial $F$ is weighted homogeneous of degree 10 with respect to the weights $w_{1}=1, w_{2}=2, w_{3}=3, w_{4}=5$. Then $H(x, y, z)=$ $x^{5} y z+y^{2} z^{2}+x^{3} y+x^{2} y^{2}+x y^{3}+y^{5}$ and $G(x, y, z)=x^{2} z+1+x^{5} y z+y^{2} z^{2}+$ $x^{3} y+x^{2} y^{2}+x y^{3}+y^{5}$. It is easily seen that $G^{-1}(0)$ is non-empty. A computer calculation shows that $\operatorname{dim} \mathbb{R}\left[x^{\prime}\right] / I(H)=140$ and $\operatorname{dim} \mathbb{R}\left[x^{\prime}\right] / I(G)=142$ and that the form $\Phi_{M}$ is non-degenerate and signature $\Phi_{M}=\chi(L)=0$.

EXAMPLE 2. Let $F_{1}(x, y, z, t, u)=x y z+x u+z^{2}+y t, F_{2}(x, y, z, t, u)=$ $u+y z+x t+x^{2} z$. The polynomials are weighted homogeneous of degrees 6 and 5 respectively, with respect to the weights $w_{1}=1, w_{2}=2, w_{3}=3, w_{4}=4$, $w_{5}=5$. Then $H_{1}(x, y, z, t)=x y z+z^{2}+y t, H_{2}(x, y, z, t)=y z+x t+x^{2} z$, $G_{1}(x, y, z, t)=x y z+x+z^{2}+y t, G_{2}(x, y, z, t)=1+y z+x t+x^{2} z$. A computer 
calculation shows that $\operatorname{dim} \mathbb{R}\left[x^{\prime}\right] / I(H)=57$ and $\operatorname{dim} \mathbb{R}\left[x^{\prime}\right] / I(G)=33$, the form $\Phi_{M}$ is non-degenerate and signature $\Phi_{M}=1$ so $\chi(L)=2$.

Acknowledgements. The author wishes to express his gratitude to Zbigniew Szafraniec for suggesting the idea of the proof, to Piotr NowakPrzygodzki for many discussions about the subject and to Andrzej Łęcki for cooperation.

\section{References}

[1] J. Damon, On the number of branches for real and complex weighted homogeneous curve singularities, Topology 30 (1991), 223-229.

[2] P. Dudziński, On topological invariants mod 2 of weighted homogeneous polynomials, ibid. 35 (1996), 323-328.

[3] H. King, thesis, Univ. of California, Berkeley, 1974.

[4] J. Milnor, Singular Points of Complex Hypersurfaces, Princeton Univ. Press, 1968.

[5] J. Milnor and P. Orlik, Isolated singularities defined by weighted homogeneous polynomials, Topology 9 (1970), 385-393.

[6] Z. Szafraniec, Topological invariants mod 2 of weighted homogeneous polynomials, Glasgow Math. J. 33 (1991), 241-245.

[7] - A formula for the Euler characteristic of a real algebraic manifold, Manuscripta Math. 85 (1994), 345-360.

[8] C. T. C. Wall, Topological invariance of the Milnor number mod 2, Topology 22 (1983), 345-350.

Institute of Mathematics

University of Gdańsk

Wita Stwosza 57

80-925 Gdańsk, Poland

E-mail: pd@delta.math.univ.gda.pl 